\title{
Neuro Fuzzy Control system for Distributed Generation Plants
}

\author{
Yury N. Bulatov ${ }^{1}$, Andrey V. Kryukov ${ }^{2}$ \\ ${ }^{1}$ Bratsk State University, \\ Bratsk, Russia \\ E-mail: bulatovyura@yandex.ru \\ ${ }^{2}$ Irkutsk State Transport University, \\ Irkutsk National Research Technical University \\ Irkutsk, Russia \\ E-mail: and_kryukov@mail.ru
}

\begin{abstract}
The transition of electric power industry to a new technological platform based on the concept of smart power grids with an active-and-adaptive network will allow to increase the efficiency of control, durability and reliability of power supply systems. The idea of applying the intelligent algorithms to control power grids becomes more pressing in case of wide using the distributed generation plants (DGP) and other grid active elements that allow to control power grid operation modes.

The article describes the DGP consisting of synchronous generators that operate in various modes and often with poor power supply quality. To provide the parallel operation stability of synchronous turbo/ hydro generators of DGP, automatic excitation regulator (AER) and automatic regulator of rotor speed (ARRS) are used. Due to low inertia constant of distributed generation unit rotors, the problem of matched tuning of AER and ARRS becomes more topical. To increase stability and reliable integration of DGP in power grids, a control system built on a neurofuzzy match system is proposed. The system corrects AER/ ARRS settings. In this case, the following smart technologies were used: genetic algorithm to search optimal settings of AER and ARRS; a neuro-fuzzy network to identify an operation mode of a DGP and power grid; fuzzy interference to correct settings of AER/ ARRS in various modes of DGP operation.

Based on modelling done in MATLAB system the efficiency of using the proposed neuro-fuzzy match system to identify DGP operation modes and to adaptively control matched tuning of AER and ARRS of synchronous generators is shown. If using the intellectual control system, one can decrease transition process time, generator voltage / frequency overcontrol, as well as provide reliability and durability of a power system in various operation modes of a DGP and power grids.
\end{abstract}

Keywords: Distributed regeneration, automatic excitation regulator, automatic regulator of rotor speed, matched tuning, neuro-fuzzy algorithms.

\section{Introduction}

Recently, the development of intelligent control algorithms and their application in contemporary electrical power systems (EPS) becomes topical. This is due to the transition of the power generation industry to a new technological platform based on the concept of intelligent EPS with an active-adaptive network [1], which provides for extensive use of active devices for regulating the modes and distributed generation (DG) plants. The industrial introduction of these technologies will improve the management efficiency, survivability and reliability of power supply systems.

The DG plants operating on the basis of synchronous turbo/ hydro generators allow obtaining a sufficiently large power and can be used to remove peak loads, stabilize voltage and frequency, and to reduce power losses in power supply systems. But in this case, parallel operation of DG plants and EPS makes the mode control issues even more complicated. Increasing the stability of parallel operation of DG plants and EPS generators, is possible using automatic excitation regulators (AER) and automatic regulator of rotor speed (ARRS). Due to low inertia constant of DG plant rotors, the problem of matched tuning of AER and ARRS becomes more topical. In addition, optimal control requires adjusting the tuning of AER and ARRS when significant changes are introduced in the operation modes of both DG plants and EPS. These requirements can be met using the use of intelligent control algorithms.

At present, a number of studies have been carried out on the use of intelligent technologies in power supply systems [1-9], and in particular, for solving the 
problems of controllers parameterization [10-12], as well as on developing controllers working on the basis of artificial intelligence technologies [13-16]. A control system that adjusts AER and ARRS tuning and built on the basis of an adaptive neuro-fuzzy inference system is proposed in this work to improve the stability and reliable integration of the DG plants in EPS. The following intelligent technologies are used in this case: the genetic algorithm (GA) to find the optimal tunings for AER and ARRS; a neuro-fuzzy network for identification of the operating mode of DG plant and EPS; a fuzzy inference for adjusting the tunings of AER and ARRS in various modes of DG plant operation.

\section{Adaptive Neuro Fuzzy Control System For Distributed Generation Plants}

For optimal control, adjustments of AER and ARRS tunings are required in case of significant changes in the operation modes of DG plants and EPS. The following is understood under such changes: significant changes in the average voltage levels in the points of DG plants connection; a large variation in the consumers load leading to noticeable variations in voltage and frequency in the attachment node of DG plants; DG plants connection and their synchronization with the network; the occurrence of emergency modes in networks and these networks topology subsequent re-configuration caused by the disconnections of the damaged elements; change of supply circuits.

A block diagram of the proposed adaptive system of DG regulators tuning control is given in fig. 1 .

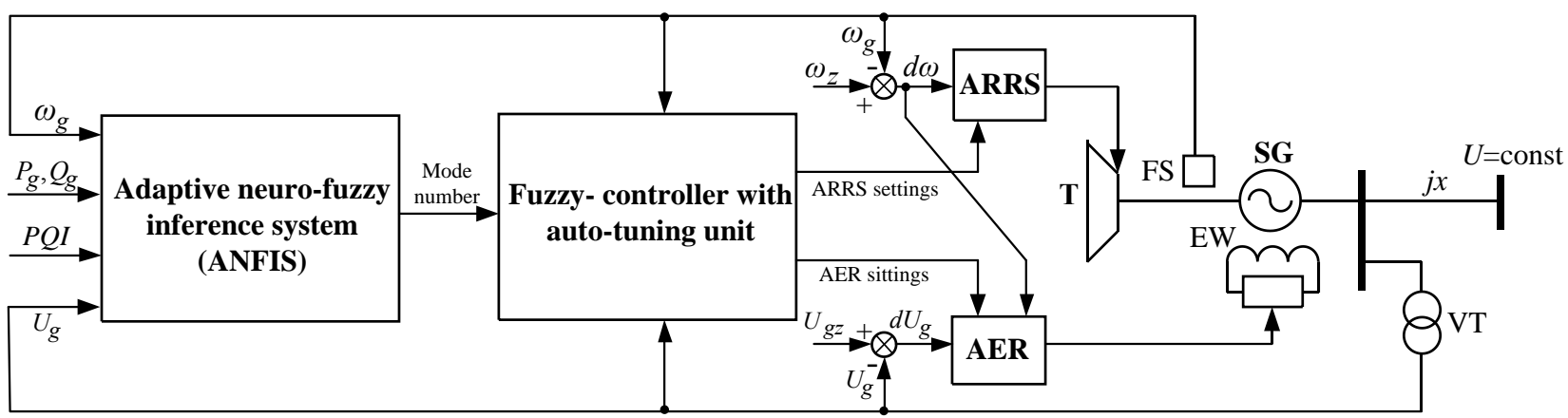

Fig. 1. Structural scheme of adaptive system for control AER and ARRS of the DG plant:

PQI - power quality indicators; FS - frequency sensor; EW - excitation winding; SG - synchronous generator; $\mathrm{T}$ - turbine; TV - voltage transformer.

The operation principle of the adaptive control system in question is to identify the operating mode of DG plant generator and to correct the current settings parameters for AER and ARRS when the operation mode is changed.

To solve the task of identifying the operation mode of DG plant generator in relation to EPS, it is suggested that an adaptive neuro-fuzzy inference system (ANFIS) should be used, which is one of the variants of hybrid neural-fuzzy networks - a neural network of a special type direct signal propagation. The architecture of such a network is isomorphic to a fuzzy knowledge base. The neuro-fuzzy networks differentiated implementation of triangular norms (multiplication and probabilistic OR) are used, as well as differentiable membership functions. This makes it possible to apply fast learning algorithms based on the method of the error back propagation for tuning the neural-fuzzy networks. In general, ANFIS implements the Sugeno fuzzy inference system in the form of a five-layer feedforward signal neural network [17].

To solve the identification problem, it is expedient to use a two-stage procedure for constructing Sugeno type fuzzy models. At the first stage, on the basis of experimental data of the voltage $\left(U_{g}\right)$, frequency $\left(\omega_{g}\right)$, power $\left(P_{g}, Q_{g}\right)$ and power quality indicators (PQI) in the connection node of DG plant together with parameters of the external network by subtractive clustering method (a generic mountain clustering method proposed by R. Jaeger and D. Filev) [18] fuzzy rules are defined. In this case, the following operation modes of DG plants generator are distinguished: unit start-up mode; idle run mode; generator autonomous operation mode for a selected load; mode of parallel operation with the network (normal steady state with different generator load); emergency mode; out-of-step mode; overload 
operation; mode of parallel operation with degraded power network and other.

At the second stage, fuzzy model parameters are tunned with ANFIS algorithm. Subtractive clustering is used here to quickly synthesize fuzzy rules from data. The result is a fuzzy model of DG plant generators operation modes which is the starting point for learning.

Fuzzy controller with automatic tuning unit is a fuzzy logic inference system with identification modules and harmonized configuring of AER and ARRS. Its input parameters are the current values of the voltage $g$ and generator rotor rotational speed ${ }^{\omega_{g}}$, as well as the operation mode identified by the ANFIS unit. The output parameters are the tuning factors of AER and ARRS, optimal for the current mode.

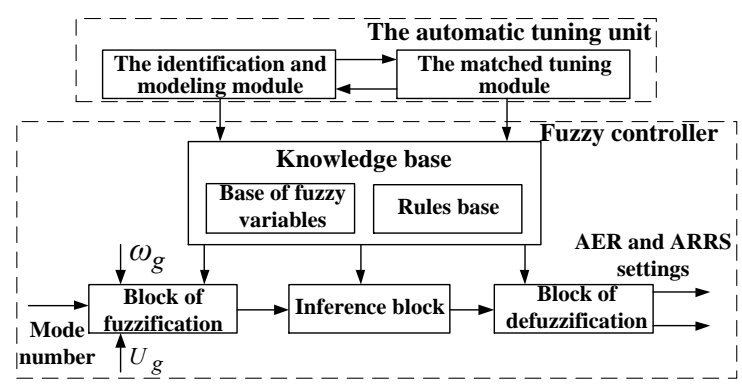

Fig. 2. The block diagram of fuzzy controller with automatic tuning unit.

The schematic block diagram of the fuzzy controller with the automatic tuning unit is shown in fig. 2.

The automatic tuning unit consists of an identification and modeling module and a harmonized configuring module, allowing it forming the fuzzy controller knowledge base.

The module of identification and modeling, based on experimental data, forms a model of the closed control system of DG plant for the current mode and transmits the received characteristic equation to the harmonized configuring module. For this purpose, nonparametric identification is used, as a result of which, on the basis of a priori information about the process, the numerical values of the complex activation factor are determined as the ratio of the spectra of the output and input signals [16]. The diagram of 'turbine-generator' closed loop system used for generation of characteristic polynomial is shown in fig. 3. Characteristic polynomial of the system under consideration is determined using the expression:

$$
D^{M}(j \omega)=\operatorname{det}\left[\mathbf{E}+\mathbf{W}_{G}(j \omega) \cdot \mathbf{W}_{R}(j \omega)\right],
$$

where $\mathrm{E}$ - a single matrix; $\mathbf{W}_{G}(j \omega)$ - matrix activation function of the tunned object (of 'turbine-generator' system) determined experimentally; $\mathbf{W}_{R}(j \omega)$ - matrix activation function of the controller considering interconnection AER and ARRS:

$$
\mathbf{W}_{R}(j \omega)=\left[\begin{array}{cc}
W_{\mathrm{ARRS}}(j \omega) & W_{\mathrm{AER}}^{\omega}(j \omega) \\
0 & W_{\mathrm{AER}}^{\mathrm{U}}(j \omega)
\end{array}\right] ; W_{\mathrm{ARRS}}(j \omega)
$$

- ARRS complex activation factor; $W_{\mathrm{AER}}^{\omega}(j \omega)$ - AER complex activation factor of the frequency-tuning channel; $W_{\mathrm{AER}}^{\mathrm{U}}(j \omega)$ - AER complex activation factor of the voltage-tuning channel.

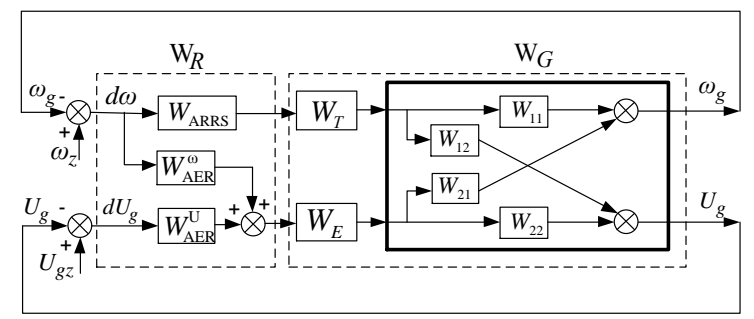

Fig. 3. T Structural scheme of the "turbine-generator" system.

The following ratios can be recorded for activation factors:

$$
\begin{aligned}
& W_{\mathrm{ARRS}}(j \omega)=\left(k_{p}+\frac{k_{i}}{0,1 j \omega}+\frac{j k_{d} \omega}{j \omega+1}\right) \cdot \frac{1}{0,01 j \omega+1} ; \\
& W_{\mathrm{AER}}^{\omega}(j \omega)=\frac{1+0,5 j \omega}{0,5 j \omega} \cdot\left[\frac{2 k_{0 \omega} j \omega}{(2 j \omega+1)(0,02 j \omega+1)}+\frac{0,05 k_{1 \omega} j \omega}{0,05 j \omega+1}\right] ; \\
& W_{\mathrm{AER}}^{\mathrm{U}}(j \omega)=\frac{1+0,5 j \omega}{0,5 j \omega} \cdot\left(k_{0 u}-\frac{0,02 k_{1 u} j \omega}{0,06 j \omega+1}\right),
\end{aligned}
$$

where $k_{p}, k i, k_{d}$-ARRS tuning factors; $k_{0 w}, k_{l w}, k_{0 \omega}$ and $k_{l \omega}-$ AER adjusting channels tuning factors.

To obtain accurate activation function for identification, it is advisable to use test broadband signals. However, interference in the operation of power supply systems is undesirable, and therefore, it is proposed to use an approach in which the controllers' user-selected noise with the use of a wavelet transform is used [16].

The harmonized configuring module allows determining the optimum tuning factors for AER and ARRS using minimization of the quality criterion based on the GA $[12,16]$ :

$$
J=\int_{0}^{\Omega} e^{2}(j \omega) d \omega \rightarrow \min ,
$$

where $\quad e(j \omega)=D^{D}(j \omega)-D^{M}(j \omega) \quad-\quad$ error between the desired and model sets of characteristic polynomials factors; $\omega$ - frequency current value from range $[0 ; \Omega]$ that determines the system 'bandwidth'. 
Butterworth or other polynomials can be used as desired polynomials.

Harmonized AER and ARRS configuring of DG plant generator is determined for all identified modes which allows creation of rule database determining fuzzy controller functioning.

It should be noted that the use of distributed generation technologies in systems with degraded power, requires the use of special active devices improving PQI $[19,20]$, for example, harmonic conditioners or DC insertion (DCI) using modern converter technologies, etc. Using the DC insertions makes allows improving the electric power quality indicators and limit the shortcircuit power on the buses of DG sources.

\section{Description of The Model and The Study Results}

The research was carried out in the MATLAB system on the model of railroad power-supply system (RPSS), the structural diagram of which is shown in fig. 4. The power supply region (PSR) of non-railway consumers was analyzed with DG plant which includes two synchronous generators with a capacity of $2.5 \mathrm{MV} \cdot \mathrm{A}$ each, supplying power to consumers with a capacity of 5 MV·A. The PSR had a microgrid cluster topology, which was implemented based on the DC insertion, and was energized from the first traction substation (TS1) or from the second (TS2) through the $6 \mathrm{kV}$ longitudinal line (fig. 4). DG plant control was carried out with the help of AER and ARRS with correction of their tuning by a fuzzy controller equipped with an automatic tuning unit. The modes were identified using the ANFIS unit.

Automatic tuning unit was used to determine AER and ARRS tuning factors for the following DG plant and external network operation modes: DG plant maximum load in free running mode; mode of DG plant and EPS parallel operation through the DC insertion (power supply from TS1); a mode of parallel operation of DG plant and EPS through the DC insertion (power supply from TS2); mode of parallel operation of DG plant and EPS through the bypass (power supply from TS1); mode of parallel operation of DG plant and EPS through the bypass (power supply from TS2).

The research was carried out for the following modes:

1. Hooking up additional loads in parallel operation mode of DG plant and EPS via DC insertion (power supply from TS1);

2. The emergence of a short circuit in the $6 \mathrm{kV}$ buses and its deenergizing by relay protection after $0.5 \mathrm{~s}$ in the parallel operation mode of DG plant and EPS through the DC insertion (power supply from TS1);

3. DC insertion deenergizing and after $0.5 \mathrm{~s}$ automatic power supply connection from EPS through bypass. In this mode, the power quality deteriorates significantly, which changes the frequency range of the system own oscillations and requires adjustments in AER and ARRS tuning, as well as DG plant power limitations.

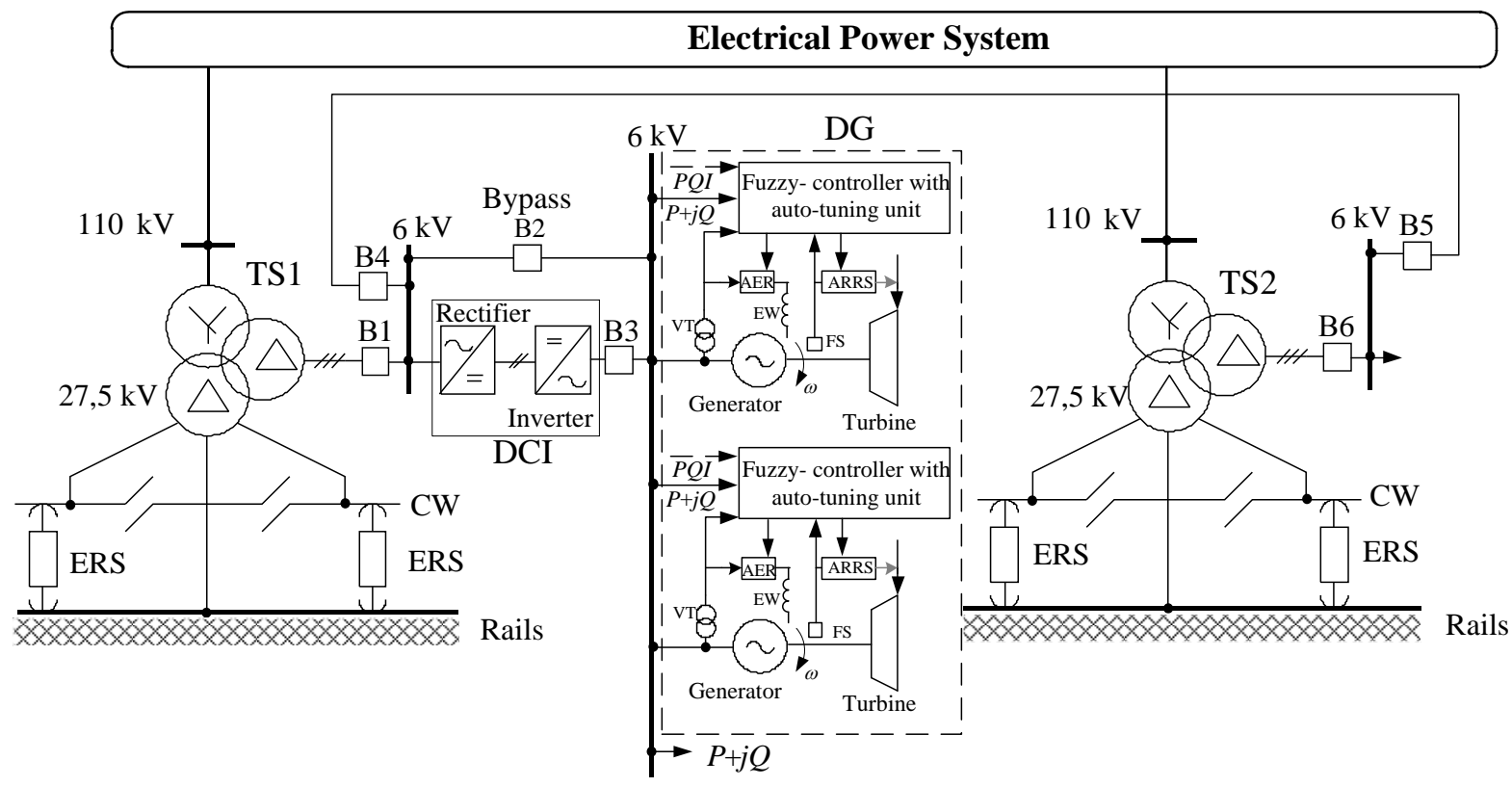

Fig. 4. Fragment of the power supply system of the railway: TS - traction substation; ERS - electro rolling stock; CW - contact wire; DCI - DC insertion; B - breaker. 
The results of computer simulation show the efficiency of the proposed adaptive control unit for tuning AER and ARRS of DG plant generator, which consists in reducing the transient time and overshooting the voltage and rotational speed of the generator rotor, and in

Voltage, rel. units

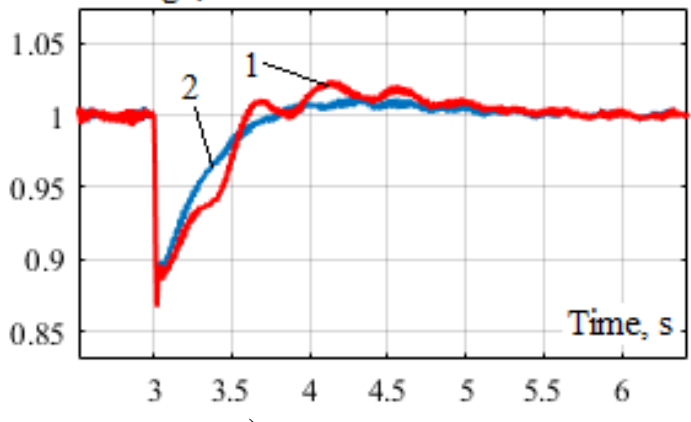

a) providing the necessary system stability margin. The oscillograms of the voltage and generator rotor speed which confirm the formulated conclusions are shown in fig. 5, 6 and 7 .

Rotor speed, rel. units

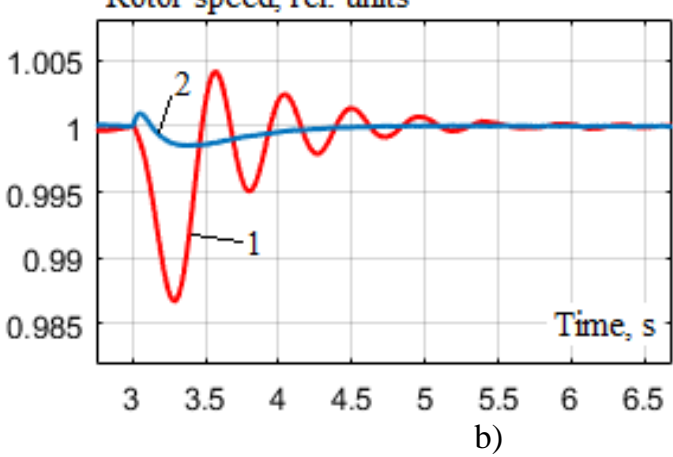

Fig. 5. Variation in the voltage (a) and frequency (b) of the generator when additional load is hooked up: 1 - without changing the tuning factors of AER and ARRS; 2 - using a fuzzy controller that changes the tunings of AER and ARRS.

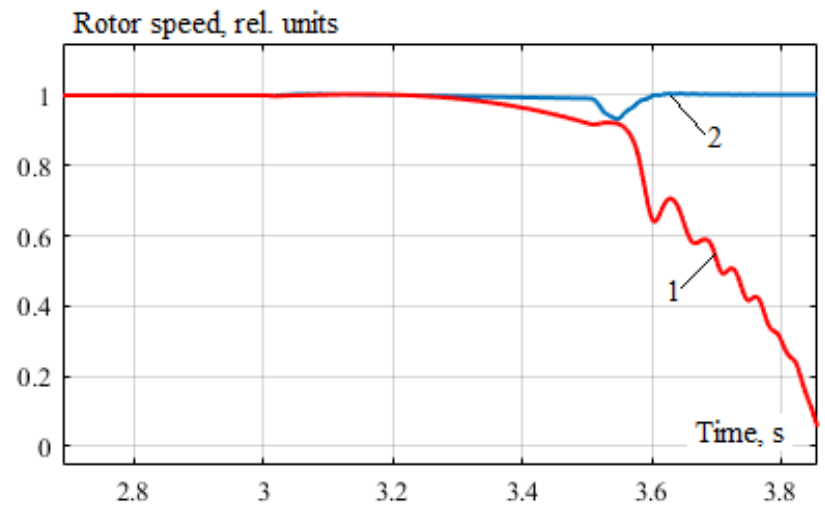

Fig. 6. Oscillograms of the generator rotor speed when a short-circuit with duration $0.5 \mathrm{~s}$ emerges on the buses of non-railway consumer at a time moment $3 \mathrm{~s}$ : 1 - without changing the tuning factors of AER and ARRS; 2 - using a fuzzy controller that changes the settings of AER and ARRS.

Voltage, $\mathrm{kV}$

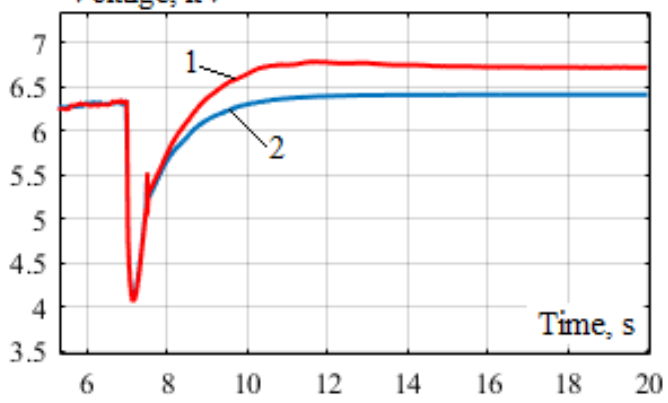

a)
Rotor speed rel. units

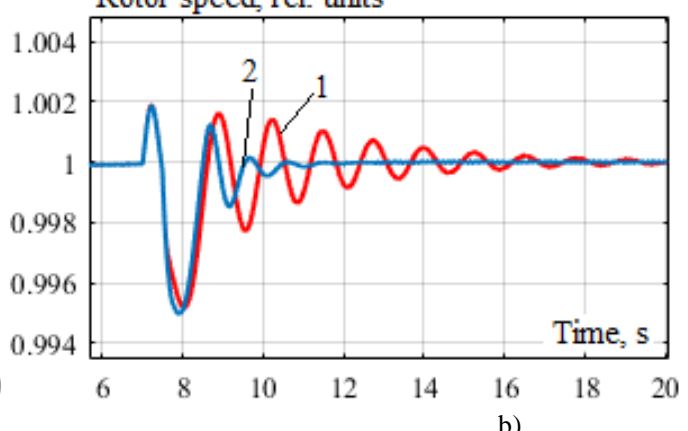

Fig. 7. The actual voltage on the tires of the non-taut consumer (a) and the rotor speed of the generator (b) when disconnecting the DCI and the connection through $0.5 \mathrm{~s}$ bypass for power supply from EPS: 1 - without changing the adjustment factors of AER and ARRS; 2 - using fuzzy-regulator, changing the settings of AER and ARRS. 
Thus, the intelligent technologies used to identify the modes and control the synchronous generator controllers using the harmonized configuring technique have made it possible to organize the knowledge base of the adaptive control system of the distributed generation plant. The intellectuality of the proposed system can be increased by more detailed ranking of DG plant possible operation in the formation of the fuzzy controller knowledge base.

\section{Conclusion}

The results of computer modelling allow to make the following conclusions:

1. An adaptive system for controlling the parameters of the distributed generation system controllers is proposed, the knowledge base of which is formed due to automatic tuning unit based on the application of the method of harmonized configuring of controllers and intelligent technologies. The use of an adaptive network based on the system of fuzzy inference and the method of subtractive clustering allows real-time identification of the DG plant generator operation mode and greatly simplifies the process of fuzzy controller rules database building.

2. The results of computer simulation allow us to make conclusion on the adaptive neural-fuzzy control unit efficiently in controlling harmonized configuring of AER and ARRS of the DG plant generator. When using an intelligent control system, the time of the transient process, the overshoot of the voltage and rotational speed of the generator rotor are reduced, and the necessary stability reserve, survivability and adaptability of the system to all possible operating modes are provided.

\section{References}

1.Morzhin Yu.I., Shakaryan Yu.G., Kucherov Yu.N. et al., Smart Grid Concept for Unified National Electrical Network of Russia, in Proceedings of IEEE PES Innovative Smart Grid Technologies Europe 2011, Manchester Dec. 5-7 2011. (Manchester, GB: IEEE, The University of Manchester, 2011) Panel session 5D. pp. 1-5.

2.Torriti J., Demand Side Management for the European Supergrid: Occupancy variances of European single-person households, Energy Policy, vol. 44, (2012) 199-206.

3.Mohsen F.N., Amin M.S., Hashim H., Application of smart power grid in developing countries, in Proc. IEEE 7th International Power Engineering and Optimization Conference (PEOCO), 2013. DOI:10.1109/PEOCO.2013.6564586.

4.Bernd M. Buchholz, Zbigniew A., Styczynski., Smart Grids Fundamentals and Technologies in Electricity Networks (Springer-Verlag Berlin Heidelberg, 2014). 396 p.

5.Wang J., Huang A.Q., Sung W., Liu Y., Baliga B.J., Smart Grid Technologies, IEEE Industrial Electronics Magazine, vol. 3. No 2. (2009) 16-23.

6.Magdi S. Mahmoud, Fouad M., AL-Sunni Control and Optimization of Distributed Generation System. (Cham: Springer International Publishing : Imprint: Springer, 2015). $578 \mathrm{p}$.

7.Martínez Ceseña E. A., Capuder T., Mancarella P., Flexible distributed multienergy generation system expansion planning under uncertainty, IEEE Transaction on Smart Grid, vol. 7, (2016) 348-357.

8.Lombardi P., Styczynski Z.A., Sokolnikova T., Suslov K., Use of energy storage in Isolated Micro Grids, in Power Systems Computation Conference (PSCC) 2014, IEEE Conference Publications. 2014, pp. 1-6. DOI: 10.1109/PSCC.2014.7038361.

9.Ph. P. Barker, R.W. De Mello, Determining the Impact of Distributed Generation on Power Systems: Part 1 - Radial Distribution Systems, in 2000 IEEE PES Summer Meeting, (Seattle, WA, USA, July 11-15) pp. 222-233.

10. Chen Y., Ma Y., Yun W., Application of Improved Genetic Algorithm in PID Controller Parameters Optimization, Telkomnika, vol. 11, no. 3, (2013) 1524-1530.

11. Jaen-Cuellar A.Y., Romero-Troncoso R. de J., MoralesVelazquez L., Osornio-Rios R.A., PID-Controller Tuning Optimization with Genetic Algorithms in Servo Systems, International Journal of Advanced Robotic Systems, vol. 10, (2013) 324-337. DOI: 10.5772/56697.

12. Bulatov Yu. N., Kryukov A. V., Optimization of automatic regulator settings of the distributed generation plants on the basis of genetic algorithm, 2nd International Conference on Industrial Engineering, Applications and Manufacturing (ICIEAM). IEEE Conference Publications. 2016, pp. 1-6. DOI: 10.1109/ICIEAM.2016.7911456.

13. Evgenije Adzic et al., Maximum Power Search in Wind Turbine Based on Fuzzy Logic Control / Evgenije Adzic, Zoran Ivanovic, Milan Adzic, Vladimir Katic, Acta Polytechnica Hungarica, vol. 6, no. 1, (2009) 131-149. 
14. Voropai N. I., Etingov P. V. Two-Stage Adaptive Fuzzy PSS Application to Power Systems, in Proc. of International Conference on Electrical Engineering ICEE'2001, July 22-26, (Xi'an, China, 2001, vol. 1) pp. 314-318.

15. Belyaev A. N., Smolovik S. V., Design of adaptive automatic excitation regulators using neuron-fuzzy modeling, Electricity, No. 3. (2002) 2-9.

16. Kryukov A. V., Kargapol'cev S. K., Bulatov Yu. N., Skrypnik O. N., Kuznetsov B. F., Intelligent control of the regulators adjustment of the distributed generation installation, Far East Journal of Electronics and Communications, Vol. 17. No. 5. (2017) 1127-1140.

17. Jang, J.-S.R., ANFIS: adaptive-network-based fuzzy inference system, IEEE Transactions on Systems, Man and Cybernetics. Vol. 23. (1993) 665-685. DOI:10.1109/21.256541

18. Yager R., Filev D., Essentials of Fuzzy Modeling and Control (USA: John Wiley \& Sons, 1984). P. 387.

19. Suslov K., Solonina N., Stepanov V., A principle of power quality control in the intelligent distribution networks, in Proc. of International symposium on smart electric distribution systems and technologies (EDST 2015) (2015) pp. 260-264.

20. Suslov K. V., Stepanov V. S., Solonina N. N., Smart grid: effect of high harmonics on electricity consumers in distribution networks, IEEE International Symposium on Electromagnetic Compatibility, 2013, pp. 841-845. 